\title{
Gold(I)-Catalyzed Domino Reaction for Furopyrans Synthesis
}

\author{
Marie Ruch, Nicolas Brach $₫$, Roméric Galéa, Patrick Wagner $₫$ and Gaëlle Blond *®i
}

Laboratoire d'Innovation Thérapeutique, Université de Strasbourg, CNRS, UMR 7200, 67000 Strasbourg, France; ruchm@unistra.fr (M.R.); nicolas.brach@etu.unistra.fr (N.B.); r.galea@unistra.fr (R.G.); pwagner@unistra.fr (P.W.)

* Correspondence: gaelle.blond@unistra.fr; Tel.: +33-368854165

Received: 12 October 2020; Accepted: 23 October 2020; Published: 27 October 2020

check for updates

\begin{abstract}
We report herein an efficient synthesis of furopyran derivatives through a gold(I)-catalyzed domino reaction. The cascade reaction starts with two regioselective cyclizations, a 5-endo-dig and a 8-endo-dig, followed with a Grob-type fragmentation and a hetero Diels-Alder. The obtained furopyran derivatives contain fused and spiro-heterocycles. During this one-pot process, four bonds and four controlled stereogenic centers including a quaternary center are formed.
\end{abstract}

Keywords: gold(I)-catalysis; domino reactions; electrocyclization; heterocycles; hetero Diels-Alder; furopyrans

\section{Introduction}

Furopyrans are an important heterocyclic motif. Among furopyran scaffolds, $4 H$-furo[2,3- $b]$ pyrans bearing fused bicyclic $\mathrm{O}, \mathrm{O}$-acetals are present in a number of biologically active products such as macrodasine A, xyloketal A, hyperaspidinol A and penicipyrone (Figure 1) [1-5].

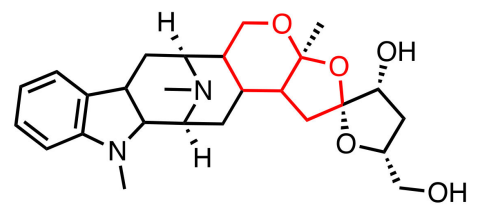

macrodasine A

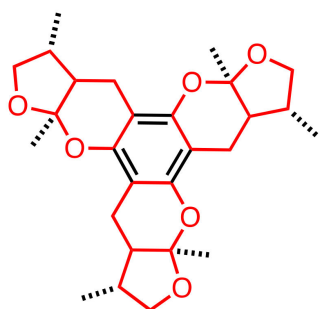

xyloketal $A$

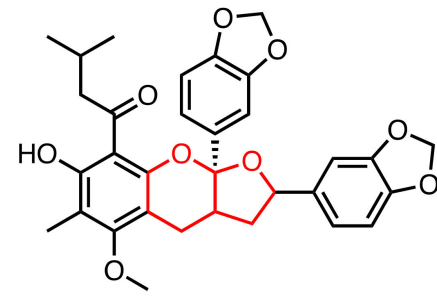

hyperaspidinol A<smiles>C/C=C/[C@H]1CO[C@H]2Oc3cc(C)oc(=O)c3CC21</smiles>

penicipyrone

Figure 1. Representative natural products with $4 H$-furo[2,3- $b$ ]pyran scaffolds.

To access a furopyran core, the hetero Diels-Alder reaction (HDA) is one of the most efficient methods, coupling a dihydrofuran with $\alpha, \beta$-unsaturated ketone [6-11]. Particularly, gold(I)-catalyzed reactions including HDA reactions have proven to be important tools for the construction of such scaffolds. Most of the gold(I)-triggered HDA reported so far rely on the in situ generation of the diene [12-14] or the dienophile [15-22]. However, a HDA between diene and dienophile generated in situ by gold-catalyzed transformations remains elusive [23].

In this context, we have previously reported the synthesis of polycyclic molecules containing furopyran cores through a gold(I)-catalyzed domino reaction (Scheme 1) [24,25]. Particularly, we have described the synthesis of two classes of furopyrans, $\mathbf{2}$ and $\mathbf{3}$, starting from the same source $\mathbf{1}$ and only changing the solvent of the reaction. 


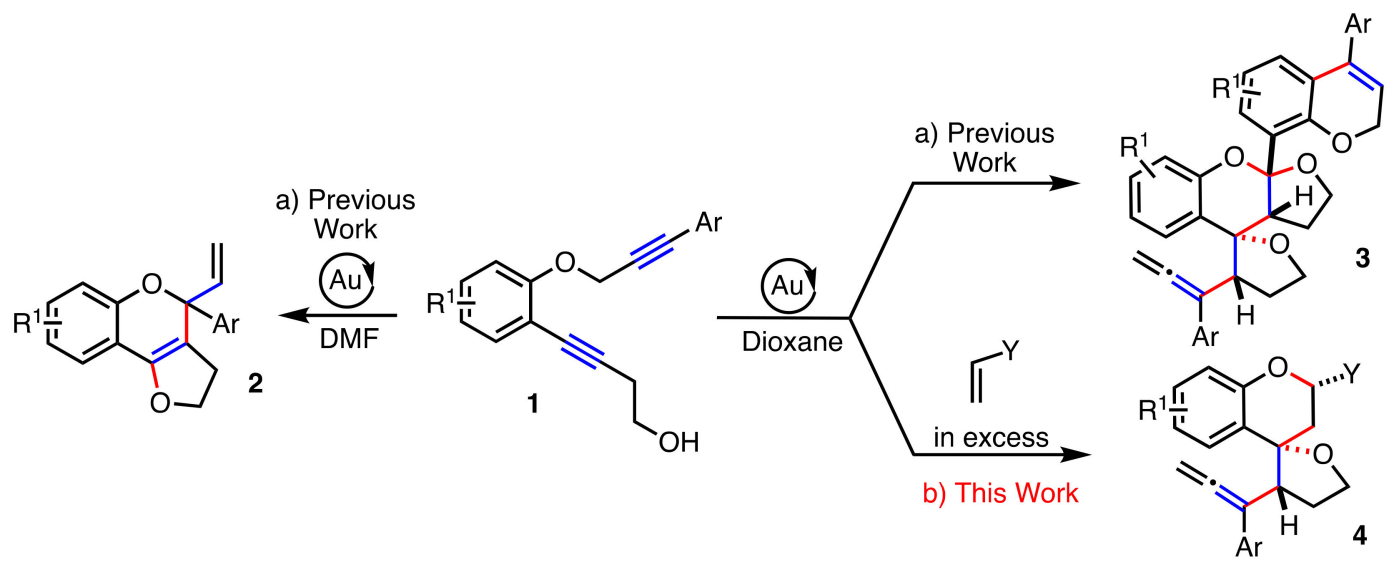

Scheme 1. Gold(I)-catalyzed cascade reaction with $\mathbf{1}$.

Indeed, in DMF, we have prepared a series of $4 H$-furo[3,2-c]pyrans 2, while in 1,4-dioxane, complex $4 H$-furo[2,3- $b$ ]pyrans 3 have been synthesized. Concerning the formation of 3 in 1,4-dioxane, the sequence includes two cyclizations: a 5-endo-dig and a regioselective 8-endo-dig cyclization giving the 8-membered ring 8 (Scheme 2). A Grob-type fragmentation allowed the formation of the diene 9 . The key step of this cascade reaction is a hetero Diels-Alder reaction (HDA) in which the formed diene $\mathbf{9}$ and the dienophile $\mathbf{1 0}$ are produced concomitantly. The formation of dienophile $\mathbf{1 0}$ comes from the protodeauration of intermediary 6 . The final gold-catalyzed hydroarylation produces 3 in good yield. Continuing these works, we have decided to introduce in the reaction mixture an excess of dienophile 11. Indeed, starting with the same starting material 1 in 1,4-dioxane, intermediate 9 will react with the excess of dienophile $\mathbf{1 1}$ instead of dienophile $\mathbf{1 0}$ also present in the reaction mixture. We describe herein the synthesis of the resulting compound 4.

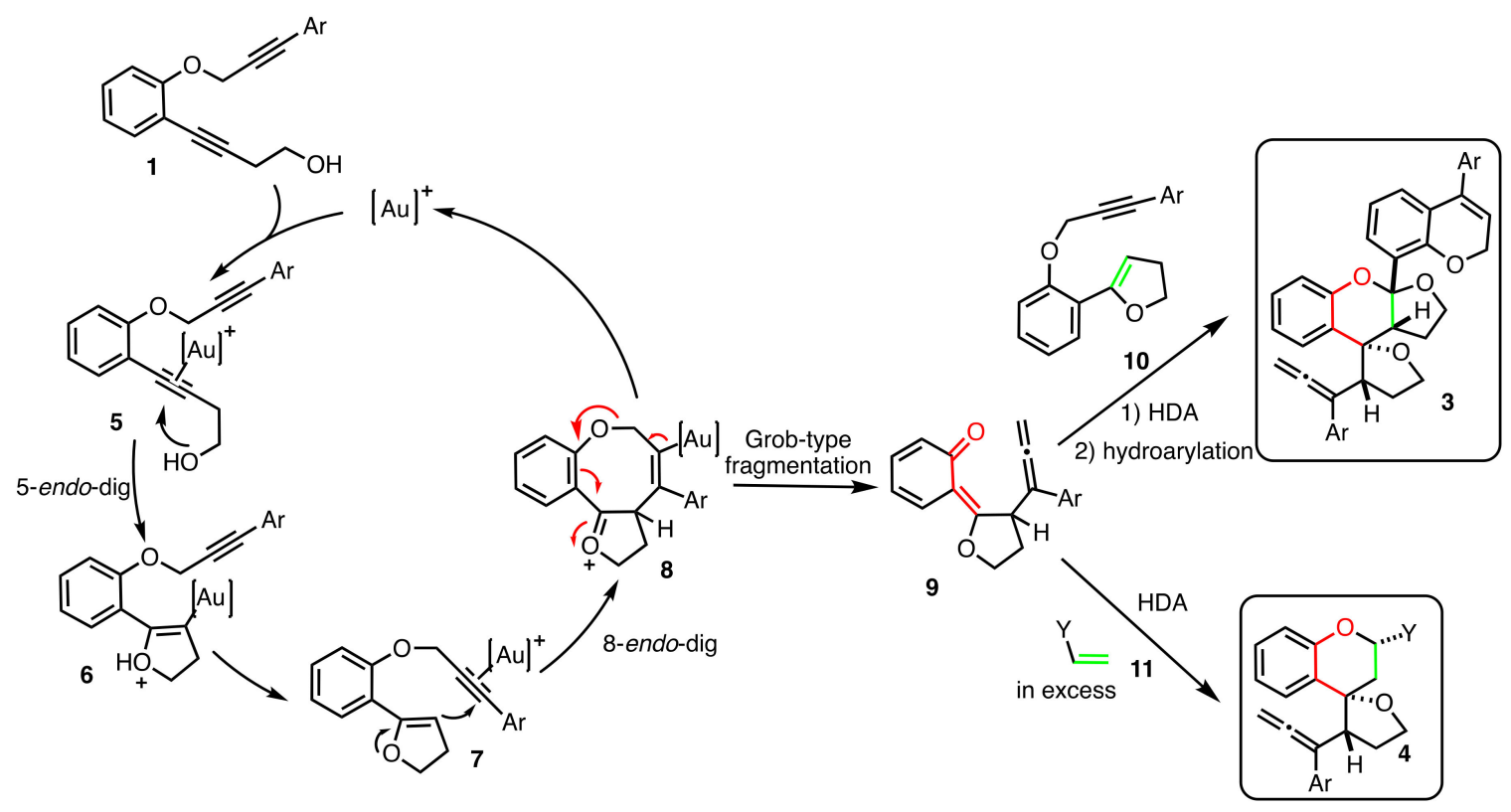

Scheme 2. Proposed mechanism.

\section{Results}

\subsection{Dienophiles Screening}

As previously described, the conditions to obtain $\mathbf{3}$ with good yield were the use of catalyst $\mathbf{A}$, in 1,4-dioxane after $20 \mathrm{~min}$ at $60^{\circ} \mathrm{C}$ under microwave irradiation. Our first investigation was the use 
of these reaction conditions in the presence of an excess of dihydrofuran; we obtained good yield of $4 \mathbf{a}(64 \%$, Entry 1, Table 1). We then decided to explore other common dienophiles [2] in order to increase the complexity of such scaffolds. The use of 4-phenylbut-3-yn-1-ol was motivated by obtaining the in situ formation of the 5-phenyl-2,3-dihydrofuran able to react as a dienophile in the reaction (entry 2) [20]. We obtained the formation of $\mathbf{5 a}$ with $7 \%$ yield. With dihydropyran, the conversion was total, and the protected alcohol $\mathbf{6}$ was not isolated completely purely (around 20\% yield) in the middle of an unidentified product (entry 3). Surprisingly, methyl or ethyl vinyl ether gave complete conversion into a complex mixture (entries 4 and 5). The same result was observed with dihydropyrrole (entry 6), phenyl vinyl thioether (entry 7) and furan-2,5-dione (entry 8).

Table 1. Optimization of the reaction conditions. ${ }^{1}$

Entry

${ }^{1}$ The reactions were performed by adding Cat. A (5 mol\%) to a solution of 1a (1equiv.) and dienophile (10 equiv.) in 1,4-dioxane $(0.145 \mathrm{M}) .{ }^{2} \mathrm{CM}$ : Complex Mixture.

\subsection{Scope of the Reaction}

As only dihydrofuran gave a good result, we investigated the scope of the reaction using dihydrofuran with various compounds of type 1 with a range of aryls exhibiting electron-donating or -withdrawing groups at the meta or para positions (Scheme 3). Our best result was obtained with the tolyl group $(4 \mathbf{b}, 80 \%)$. A para- or meta-methoxy group on the aryl ring made the reaction possible 
and $4 \mathrm{c}$ and $4 \mathrm{~d}$ were isolated with $60 \%$ yield. Results are more disparate with halogen substitutions, where yields between 28 to $70 \%$ were obtained (4e-4i). As described previously, with ortho-substituted aryl groups, the steric hindrance hampers the reaction. Indeed, with an ortho-chloro substituted aryl, less than $5 \%$ of 4 was observed. With aryls exhibiting electron-withdrawing groups such as $\mathrm{CF}_{3}$ and $\mathrm{NO}_{2}$, yields between 34 and $60 \%(4 \mathbf{j}-41)$ were obtained. Heteroaromatics such as thiophene and benzothiophene gave good results ( $4 m-4 n$, more than $65 \%)$.
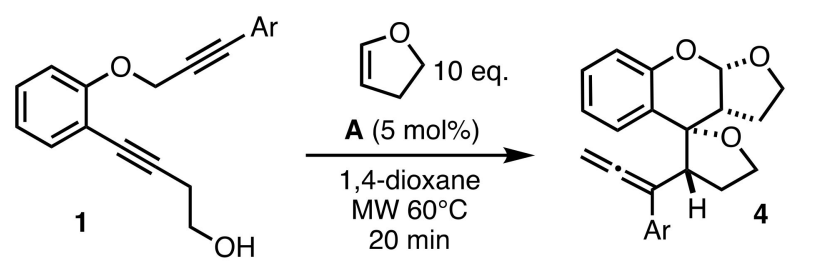

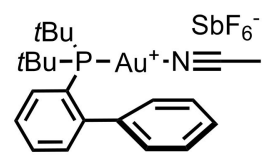

Cat. A<smiles>C=C=C(c1ccccc1)[C@H]1CCO[C@@H]2CCO[C@@H]2c2ccccc2O1</smiles><smiles>C=C(C(=O)O[Na])[C@]12CCO[C@H]1[C@@H]1OCC[C@H]1Oc1ccccc12</smiles><smiles>C=C=C(c1cccc(OC)c1)[C@@H]1CCO[C@]12c1ccccc1O[C@H]1OCC[C@@H]12</smiles><smiles>C=C(c1ccc(OC)cc1)[C@H]1CCO[C@]12c1ccccc1O[C@H]1OCC[C@@H]12</smiles><smiles>C=C(c1cccc(F)c1)[C@H]1CCO[C@]12c1ccccc1O[C@H]1OCC[C@@H]12</smiles><smiles>C=C=C(c1cccc(Br)c1)[C@@H]1CCO[C@H]1[C@@H]1CCO[C@H]1Oc1ccccc1O[Na]</smiles><smiles>C=C(c1ccc(F)c(Cl)c1)[C@H]1CCO[C@]12c1ccccc1O[C@H]1OCC[C@@H]12</smiles><smiles>C=C=C(c1ccc(Br)c(F)c1)[C@H]1CCO[C@]12c1ccccc1O[C@H]1OCC[C@@H]12</smiles><smiles>C=C=C(c1ccc(Cl)cc1)[C@H]1CCO[C@]12c1ccccc1O[C@H]1OCC[C@@H]12</smiles><smiles>C=C=C(c1ccccc1Cl)[C@H]1CCO[C@]12c1ccccc1O[C@H]1OCC[C@@H]12</smiles><smiles>C=C(c1ccc(C(F)(F)F)cc1)[C@H]1CCO[C@]12c1ccccc1O[C@H]1OCC[C@@H]12</smiles><smiles>C=C=C(c1ccc([N+](=O)[O-])cc1)[C@H]1CCO[C@]12c1ccccc1O[C@H]1OCC[C@@H]12</smiles><smiles>C=C(C(=C)[C@H]1CCO[C@]12c1ccccc1O[C@H]1OCC[C@@H]12)c1cccc([N+](=O)[O-])c1</smiles><smiles>C=C=C(c1cccs1)[C@@H]1CCO[C@]12c1ccccc1O[C@H]1OCC[C@@H]12</smiles><smiles>C=C(c1cc2ccccc2s1)[C@H]1CCO[C@]12c1ccccc1O[C@H]1OCC[C@@H]12</smiles>

Scheme 3. Scope of the reaction.

\section{Conclusions}

In conclusion, we have described the synthesis of new $4 \mathrm{H}$-furo[2,3-b]pyrans 4 via a gold-catalyzed domino reaction. The sequence includes two regioselective cyclizations, a 5-endo-dig and a 8-endo-dig, followed with a Grob-type fragmentation and a hetero Diels-Alder reaction. A total of 14 compounds have been described and isolated, with yields ranging between $28-80 \%$.

\section{Materials and Methods}

\subsection{General Information}

All reagents, chemicals and dry solvents were purchased from commercial sources and used without purification. When mentioned that the reaction was conducted in dry media, glassware dried for several hours at $110^{\circ} \mathrm{C}$ in an oven was used. Triethylamine $\left(\mathrm{Et}_{3} \mathrm{~N}\right)$ and diisopropylamine (DIPA) were distilled from $\mathrm{KOH}$ in an S-tube prior to each experiment in which they were involved. Reactions 
were monitored by TLC (thin layer silica gel chromatography) using Merck silica gel 60 F254 on aluminum sheets. TLC plates were visualized under UV light and revealed with acidic $p$-anisaldehyde stain or $\mathrm{KMnO}_{4}$ stain. Crude products were purified by flash column chromatography on Merck silica gel Si $60(40-63 \mu \mathrm{m})$. NMR spectra were recorded in $\mathrm{CDCl}_{3}$ on a Bruker Avance III BBFO+ probe spectrometer $400 \mathrm{MHz}$ for ${ }^{1} \mathrm{H}$ analyses and $100 \mathrm{MHz}$ for ${ }^{13} \mathrm{C}$ analyses. Proton chemical shifts are reported in ppm ( $\delta)$, relatively to residual $\mathrm{CHCl}_{3}(\delta 7.26 \mathrm{ppm})$. Multiplicities are reported as follows: singlet (s), doublet (d), triplet (t), quartet (q), quintet (quint), broad singlet (bs), broad doublet (bd) combinations or multiplet (m). Coupling constant values J are given in Hz. Carbon chemical shifts are reported in ppm ( $\delta)$, relative to the internal standard $\mathrm{CDCl}_{3}(\delta 77.23 \mathrm{ppm}) .{ }^{1} \mathrm{H}$ and ${ }^{13} \mathrm{C}$ NMR signals were assigned mostly on the basis of 2D-NMR (COSY, HSQC, HMBC) experiments. High resolution mass spectral analyses (HRMS) were performed using an Agilent 1200 RRLC HPLC chain and an Agilent 6520 Accurate mass QToF. Infrared spectra (IR) were recorded on a FTIR Thermo Nicollet ATR 380, Diamant Spectrometer.

All compounds $\mathbf{1 a}-1 \mathbf{e}$ and $\mathbf{1 h}-\mathbf{1} \mathbf{n}$ have been already described [6].

\subsection{Synthesis of $\mathbf{1}$ and $\mathbf{1 g}$}

Anhydrous THF and distilled $\mathrm{Et}_{3} \mathrm{~N}$ were mixed in a 2-necked flask under argon. The iodoaryl (1.5 eq.), $\mathrm{PdCl}_{2}\left(\mathrm{PPh}_{3}\right)$ (0.03 eq.) and $\mathrm{CuI}(0.06$ eq.) were added to the flask and this mixture was degassed with argon for $15 \mathrm{~min}$. The alkyne (1 eq.) was dissolved in THF degassed with argon for $15 \mathrm{~min}$ and added to the 2-necked flask. The mixture was stirred overnight at room temperature $\left(20^{\circ} \mathrm{C}\right)$ and monitored by TLC $\left(9 / 1\right.$ pent/Et $\left.t_{2} \mathrm{O}\right)$. Once the TLC showed complete conversion of the true alkyne, the reaction mixture was filtered through a pad of Celite with $\mathrm{CH}_{2} \mathrm{Cl}_{2}$ as eluent and concentrated to give the crude product as a dark-brown solid. The latter was purified by flash column chromatography (98/2 pent/ $\mathrm{Et}_{2} \mathrm{O}$ ) to afford the pure tert-butyldimethylsilyl-protected coupling product. TBAF (1 eq.) was added to a solution of this latter protected product in THF at $0{ }^{\circ} \mathrm{C}$. This mixture was stirred at room temperature until the TLC $\left(9 / 1\right.$ pent/ $\left./ \mathrm{Et}_{2} \mathrm{O}\right)$ showed complete conversion of the starting material. The reaction mixture was dissolved in a saturated aqueous $\mathrm{NH}_{4} \mathrm{Cl}$ solution. The aqueous phase was extracted with $\mathrm{CH}_{2} \mathrm{Cl}_{2}$. The gathered organic layers were dried over $\mathrm{MgSO}_{4}$, filtered and concentrated to afford the crude as a yellowish oil. The latter was purified by flash column chromatography $\left(6 / 4\right.$ pent $\left./ \mathrm{Et}_{2} \mathrm{O}\right)$ to afford the pure deprotected compound (1f and $\left.\mathbf{1 g}\right)$.

\subsubsection{Synthesis of 4-(2-((3-(3-bromophenyl)prop-2-yn-1-yl)oxy)phenyl)but-3-yn-1-ol 1f}

Compound $1 \mathrm{f}$ was prepared following the general procedure using alkyne (500 $\mathrm{mg}, 1.6 \mathrm{mmol}$, 1 eq.), THF (10 mL), $\mathrm{PdCl}_{2}\left(\mathrm{PPh}_{3}\right)_{2}$ (34 mg, $0.05 \mathrm{mmol}, 0.03$ eq.), CuI (18 mg, 0,1 mmol, 0.06 eq.), 3-bromoiodobenzene $(679 \mathrm{mg}, 2.4 \mathrm{mmol}, 1.5 \mathrm{eq}),. \mathrm{Et}_{3} \mathrm{~N}(4 \mathrm{~mL})$ and TBAF (1.6 mL, $1.60 \mathrm{mmol}$, 1 eq.) in THF $(10 \mathrm{~mL})$ for the deprotection. Purification by chromatography on silica gel afforded compound 1f $\left(81 \%, 499 \mathrm{mg}, 1.405 \mathrm{mmol}\right.$ in two steps) as an orange oil. ${ }^{1} \mathrm{H}$ NMR $(400 \mathrm{MHz}$, $\left.\mathrm{CDCl}_{3}\right) \delta 7.67(\mathrm{t}, J=1.7 \mathrm{~Hz}, 1 \mathrm{H}), 7.55(\mathrm{ddd}, J=8.0,2.1,1.1 \mathrm{~Hz}, 1 \mathrm{H}), 7.50(\mathrm{dd}, J=7.6,1.7 \mathrm{~Hz}, 1 \mathrm{H})$, $7.45(\mathrm{dt}, J=7.8,1.3 \mathrm{~Hz}, 1 \mathrm{H}), 7.38(\mathrm{ddd}, J=8.4,7.5,1.8 \mathrm{~Hz}, 1 \mathrm{H}), 7.26(\mathrm{t}, J=7.9 \mathrm{~Hz}, 1 \mathrm{H}), 7.15(\mathrm{dd}, J=8.4$, $1.1 \mathrm{~Hz}, 1 \mathrm{H}), 7.05(\mathrm{td}, J=7.5,1.0 \mathrm{~Hz}, 1 \mathrm{H}), 5.07(\mathrm{~s}, 2 \mathrm{H}), 3.92(\mathrm{q}, J=5.2 \mathrm{~Hz}, 2 \mathrm{H}), 2.84(\mathrm{t}, J=6.1 \mathrm{~Hz}, 2 \mathrm{H})$, 2.35 (s, 1H). ${ }^{13} \mathrm{C}$ NMR $\left(101 \mathrm{MHz}, \mathrm{CDCl}_{3}\right) \delta 158.3,134.6,133.6,132.0,130.5,129.9,129.3,124.3,122.2$, 121.6, 113.5, 112.9, 91.3, 86.1, 85.1, 78.9, 61.2, 57.4, 24.4. HRMS ESI: Calculated for $\mathrm{C}_{19} \mathrm{H}_{15} \mathrm{BrO}_{2}[\mathrm{M}+\mathrm{H}]^{+}$ 355.0334, found 355.0311 (Diff.: 4.58 ppm).

\subsubsection{4-(2-((3-(3-chloro-4-fluorophenyl)prop-2-yn-1-yl)oxy)phenyl)but-3-yn-1-ol 19}

Compound $1 \mathrm{~g}$ was prepared following the general procedure using alkyne (500 $\mathrm{mg}, 1.6 \mathrm{mmol}$, 1 eq.), THF (10 mL), $\mathrm{PdCl}_{2}\left(\mathrm{PPh}_{3}\right)_{2}$ (34 mg, $0.05 \mathrm{mmol}, 0.03$ eq.), CuI (18 mg, 0,1 mmol, 0.06 eq.), 3-chloro-4-chloroiodobenzene ( $615 \mathrm{mg}, 2.4 \mathrm{mmol}, 1.5 \mathrm{eq}$.), $\mathrm{Et}_{3} \mathrm{~N}(4 \mathrm{~mL})$ and TBAF ( $0.86 \mathrm{~mL}, 0.86 \mathrm{mmol}$, 1 eq.) in THF $(10 \mathrm{~mL})$ for the deprotection. Purification by chromatography on silica gel afforded compound $1 \mathrm{~g}\left(72 \%, 380 \mathrm{mg}, 1.15 \mathrm{mmol}\right.$ in two steps) of as an orange oil. ${ }^{1} \mathrm{H}$ NMR $\left(400 \mathrm{MHz}, \mathrm{CDCl}_{3}\right)$ 
$\delta 7.67(\mathrm{dd}, J=7.0,2.1 \mathrm{~Hz}, 1 \mathrm{H}), 7.60(\mathrm{dd}, J=7.6,1.7 \mathrm{~Hz}, 1 \mathrm{H}), 7.54-7.44(\mathrm{~m}, 2 \mathrm{H}), 7.30-7.20(\mathrm{~m}, 2 \mathrm{H})$, $7.15(\mathrm{td}, J=7.5,1.0 \mathrm{~Hz}, 1 \mathrm{H}), 5.15(\mathrm{~s}, 2 \mathrm{H}), 4.02(\mathrm{t}, J=6.1 \mathrm{~Hz}, 2 \mathrm{H}), 2.94(\mathrm{t}, J=6.1 \mathrm{~Hz}, 2 \mathrm{H}), 2.49(\mathrm{~s}, 1 \mathrm{H})$. ${ }^{13} \mathrm{C}$ NMR $\left(101 \mathrm{MHz}, \mathrm{CDCl}_{3}\right) \delta 158.4(\mathrm{~d}, J=252.5 \mathrm{~Hz}), 158.3,134.1,133.6,131.9(\mathrm{~d}, J=7.0 \mathrm{~Hz}), 129.3$, 121.6, $121.3(\mathrm{~d}, J=18.0 \mathrm{~Hz}), 119.5(\mathrm{~d}, J=4.0 \mathrm{~Hz}), 116.8(\mathrm{~d}, J=21.0 \mathrm{~Hz}), 113.5,112.9,91.3,85.4,84.6$, 78.9, 61.1, 57.3, 24.3. HRMS ESI: Calculated for $\mathrm{C}_{19} \mathrm{H}_{14} \mathrm{ClFO}_{2}[\mathrm{M}+\mathrm{H}]^{+} 329.0745$, found 329.0723 (Diff.: 4.91 ppm).

\subsection{General Procedure for Gold(I) Catalyzed Cascade Reactions: Preparation of $4 \boldsymbol{a}-\boldsymbol{n}$}

Substrate 1 (1 eq.) and dihydrofuran (10 eq.) were placed in a 0.5-2 mL microwave reactor and dissolved in anhydrous 1,4-dioxane. Catalyst A (0.05 eq.) was added into the reactor. Once all the reagents were dissolved, the reactor was placed into the microwave for $20 \mathrm{~min}$ at $60^{\circ} \mathrm{C}$. The reaction mixture was filtered through a pad of Celite with $\mathrm{CH}_{2} \mathrm{Cl}_{2}$ as eluent. After solvent evaporation under reduced pressure, purification of the crude by flash column chromatography provided the furopyran adduct 4 .

4.3.1. Synthesis of (2S,3R,3a'R,9a'S)-3-(1-phenylpropa-1,2-dien-1-yl)-2' $, 3^{\prime}, 3 a^{\prime}, 4,5,9 a^{\prime}$-hexahydro-3Hspiro[furan-2,4'-furo[2,3-b]chromene] 4a

Compound 4a was prepared following the general procedure using compound 1a $(20 \mathrm{mg}$, $0.072 \mathrm{mmol}, 1$ eq.), dihydrofuran (51 $\mathrm{mg}, 0.72 \mathrm{mmol}, 10$ eq.) and catalyst $\mathbf{A}(3 \mathrm{mg}, 0.004 \mathrm{mmol}$, 0.05 eq.) in 1,4-dioxane $(0.4 \mathrm{~mL})$. Purification by chromatography on silica gel $\left(8 / 2\right.$ pentane/ $\left./ \mathrm{t}_{2} \mathrm{O}\right)$ afforded compound $4 \mathrm{a}(64 \%, 16 \mathrm{mg}, 0.046 \mathrm{mmol})$ as an amorphous white solid. ${ }^{1} \mathrm{H}$ NMR $(400 \mathrm{MHz}$, $\left.\mathrm{CDCl}_{3}\right) \delta 7.28-7.24(\mathrm{~m}, 1 \mathrm{H}), 7.23-7.11(\mathrm{~m}, 5 \mathrm{H}), 7.11-7.05(\mathrm{~m}, 1 \mathrm{H}), 6.89(\mathrm{td}, J=7.5,1.2 \mathrm{~Hz}, 1 \mathrm{H})$, $6.62(\mathrm{dd}, J=8.1,1.2 \mathrm{~Hz}, 1 \mathrm{H}), 5.31(\mathrm{~d}, J=5.6 \mathrm{~Hz}, 1 \mathrm{H}), 4.81(\mathrm{dd}, J=11.7,1.3 \mathrm{~Hz}, 1 \mathrm{H}), 4.42(\mathrm{~d}, J=11.7 \mathrm{~Hz}$, $1 \mathrm{H}), 4.33(\mathrm{td}, J=8.4,3.8 \mathrm{~Hz}, 1 \mathrm{H}), 4.10(\mathrm{td}, J=8.4,7.3 \mathrm{~Hz}, 1 \mathrm{H}), 3.87-3.73(\mathrm{~m}, 2 \mathrm{H}), 3.48-3.37(\mathrm{~m}, 1 \mathrm{H})$, $2.75(\mathrm{td}, J=9.4,5.6 \mathrm{~Hz}, 1 \mathrm{H}), 2.48(\mathrm{dtd}, J=12.8,7.3,3.8 \mathrm{~Hz}, 1 \mathrm{H}), 2.30(\mathrm{dq}, J=12.7,8.5 \mathrm{~Hz}, 1 \mathrm{H}), 2.13-1.99$ $(\mathrm{m}, 1 \mathrm{H}), 1.70(\mathrm{dtd}, J=12.9,9.6,8.4 \mathrm{~Hz}, 1 \mathrm{H}) .{ }^{13} \mathrm{C} \mathrm{NMR}\left(101 \mathrm{MHz}, \mathrm{CDCl}_{3}\right) \delta 209.3,153.0,137.0,129.0$, 128.4, 127.5, 126.8, 126.5, 125.2, 120.8, 116.0, 105.1, 101.6, 85.0, 78.3, 67.8, 67.0, 49.7, 49.6, 33.3, 27.0. HRMS ESI: Calculated for $\mathrm{C}_{23} \mathrm{H}_{23} \mathrm{O}_{3}[\mathrm{M}+\mathrm{H}]^{+} 347.1647$, found 347.1642 (Diff.: 0.31 ppm).

4.3.2. Synthesis of (2S,3R,3a'R,9a'S)-3-(1-(p-tolyl)propa-1,2-dien-1-yl)-2' $3^{\prime}, 3 \mathrm{a}^{\prime}, 4,5,9 \mathrm{a}^{\prime}$-hexahydro-3Hspiro[furan-2,4'-furo[2,3-b]chromene] $4 \mathrm{~b}$

Compound $\mathbf{4 b}$ was prepared following the general procedure using compound $\mathbf{1 b}(50 \mathrm{mg}$, $0.172 \mathrm{mmol}, 1$ eq.), dihydrofuran $(120 \mathrm{mg}, 1.72 \mathrm{mmol}, 10$ eq.) and catalyst $\mathbf{A}(7 \mathrm{mg}, 0.009 \mathrm{mmol}$, 0.05 eq.) in 1,4-dioxane $(0.4 \mathrm{~mL})$. Purification by chromatography on silica gel $\left(8 / 2\right.$ pentane $\left./ \mathrm{Et}_{2} \mathrm{O}\right)$ afforded compound $4 \mathbf{b}(80 \%, 50 \mathrm{mg}, 0.138 \mathrm{mmol})$ as an orange oil. ${ }^{1} \mathrm{H} \mathrm{NMR}\left(400 \mathrm{MHz}, \mathrm{CDCl}_{3}\right) \delta 7.24$ $(\mathrm{dd}, J=7.7,1.6 \mathrm{~Hz}, 1 \mathrm{H}), 7.10(\mathrm{td}, J=7.7,1.7 \mathrm{~Hz}, 1 \mathrm{H}), 7.05(\mathrm{~d}, J=8.4 \mathrm{~Hz}, 2 \mathrm{H}), 7.01(\mathrm{~d}, J=8.4 \mathrm{~Hz}, 2 \mathrm{H})$, $6.90(\mathrm{dd}, J=7.5,1.2 \mathrm{~Hz}, 1 \mathrm{H}), 6.64(\mathrm{dd}, J=8.1,1.2 \mathrm{~Hz}, 1 \mathrm{H}), 5.25(\mathrm{~d}, J=5.7 \mathrm{~Hz}, 1 \mathrm{H}), 4.76(\mathrm{~d}, J=11.5 \mathrm{~Hz}, 1 \mathrm{H})$, $4.32(\mathrm{td}, J=8.8,3.6 \mathrm{~Hz}, 2 \mathrm{H}), 4.09(\mathrm{td}, J=8.7,7.2 \mathrm{~Hz}, 1 \mathrm{H}), 3.88-3.70(\mathrm{~m}, 2 \mathrm{H}), 3.38(\mathrm{dd}, J=8.9,7.1 \mathrm{~Hz}, 1 \mathrm{H})$, $2.72(\mathrm{td}, J=9.5,5.6 \mathrm{~Hz}, 1 \mathrm{H}), 2.44(\mathrm{dtd}, J=12.7,7.1,3.5 \mathrm{~Hz}, 1 \mathrm{H}), 2.38-2.22(\mathrm{~m}, 4 \mathrm{H}), 2.15-1.99(\mathrm{~m}, 1 \mathrm{H})$, $1.70(\mathrm{dq}, J=12.8,8.9 \mathrm{~Hz}, 1 \mathrm{H}) .{ }^{13} \mathrm{C}$ NMR $\left(101 \mathrm{MHz}, \mathrm{CDCl}_{3}\right) \delta 209.1,153.0,136.5,134.1,129.1,129.0$, 127.6, 126.3, 125.2, 120.7, 116.0, 104.6, 101.5, 85.0, 78.1, 67.7, 67.0, 49.9, 49.8, 33.3, 27.0, 21.1. HRMS ESI: Calculated for $\mathrm{C}_{24} \mathrm{H}_{25} \mathrm{O}_{3}[\mathrm{M}+\mathrm{H}]^{+}$361.1804, found 361.1793 (Diff.: 1.59 ppm).

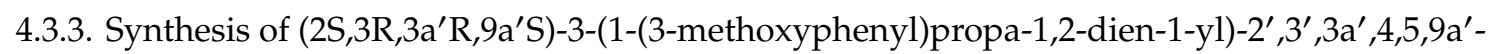
hexahydro-3H-spiro[furan-2,4'-furo[2,3-b]chromene] 4c

Compound $4 \mathbf{c}$ was prepared following the general procedure using compound $1 \mathrm{c}$ (51 $\mathrm{mg}$, $0.167 \mathrm{mmol}, 1$ eq.), dihydrofuran $(117 \mathrm{mg}, 1.67 \mathrm{mmol}, 10$ eq.) and catalyst $\mathbf{A}(6 \mathrm{mg}, 0.008 \mathrm{mmol}$, 0.05 eq.) in 1,4-dioxane $(1 \mathrm{~mL})$. Purification by chromatography on silica gel $\left(8 / 2\right.$ pentane $\left./ \mathrm{Et}_{2} \mathrm{O}\right)$ afforded compound $4 \mathbf{c}(61 \%, 39 \mathrm{mg}, 0.102 \mathrm{mmol})$ as an amorphous yellow solid. ${ }^{1} \mathrm{H}$ NMR $(400 \mathrm{MHz}$, $\left.\mathrm{CDCl}_{3}\right) \delta 7.29-7.20(\mathrm{~m}, 1 \mathrm{H}), 7.16-7.05(\mathrm{~m}, 2 \mathrm{H}), 6.88(\mathrm{td}, J=7.5,1.2 \mathrm{~Hz}, 1 \mathrm{H}), 6.78(\mathrm{dt}, J=7.9,1.2 \mathrm{~Hz}$, 
$1 \mathrm{H}), 6.70-6.59(\mathrm{~m}, 3 \mathrm{H}), 5.35(\mathrm{~d}, J=5.7 \mathrm{~Hz}, 1 \mathrm{H}), 4.81(\mathrm{dd}, J=11.7,1.4 \mathrm{~Hz}, 1 \mathrm{H}), 4.52-4.37(\mathrm{~m}, 1 \mathrm{H})$, $4.31(\mathrm{td}, J=8.4,3.9 \mathrm{~Hz}, 1 \mathrm{H}), 4.08(\mathrm{td}, J=8.4,7.4 \mathrm{~Hz}, 1 \mathrm{H}), 3.94-3.65(\mathrm{~m}, 5 \mathrm{H}), 3.47-3.26(\mathrm{~m}, 1 \mathrm{H})$, $2.75(\mathrm{td}, J=9.5,5.7 \mathrm{~Hz}, 1 \mathrm{H}), 2.46(\mathrm{dtd}, J=12.7,7.3,3.9 \mathrm{~Hz}, 1 \mathrm{H}), 2.28(\mathrm{dq}, J=12.6,8.4 \mathrm{~Hz}, 1 \mathrm{H})$, 2.05 (dddd, $J=12.9,9.2,7.2,4.8 \mathrm{~Hz}, 1 \mathrm{H}), 1.69$ (ddt, $J=12.9,9.9,8.7 \mathrm{~Hz}, 1 \mathrm{H}) .{ }^{13} \mathrm{C}$ NMR $(101 \mathrm{MHz}$, $\left.\mathrm{CDCl}_{3}\right) \delta 209.2,159.6,153.0,138.5,129.2,129.0,127.5,125.1,120.7,118.8,116.0,112.4,112.3,105.2,101.6$, 84.9, 78.3, 67.8, 66.9, 55.2, 49.6, 49.6, 33.3, 27.0. HRMS ESI: Calculated for $\mathrm{C}_{24} \mathrm{H}_{25} \mathrm{O}_{4}[\mathrm{M}+\mathrm{H}]^{+} 377.1753$, found 377.1750 (Diff.: $-0.74 \mathrm{ppm}$ ).

4.3.4. Synthesis of (2S,3R,3a'R,9a'S)-3-(1-(4-methoxyphenyl)propa-1,2-dien-1-yl)-2', 3',3a',4,5,9a'hexahydro-3H-spiro[furan-2,4'-furo[2,3-b]chromene] $4 \mathrm{~d}$

Compound $4 \mathbf{d}$ was prepared following the general procedure using compound $\mathbf{1 d}(49 \mathrm{mg}$, $0.160 \mathrm{mmol}, 1$ eq.), dihydrofuran (112 $\mathrm{mg}, 1.60 \mathrm{mmol}, 10$ eq.) and catalyst $\mathbf{A}(7 \mathrm{mg}, 0.009 \mathrm{mmol}$, 0.05 eq.) in 1,4-dioxane ( $1 \mathrm{~mL}$ ). Purification by chromatography on silica gel $\left(8 / 2\right.$ pentane/ $\left./ \mathrm{E}_{2} \mathrm{O}\right)$ afforded compound $4 \mathrm{~d}(60 \%, 37 \mathrm{mg}, 0.098 \mathrm{mmol})$ as a yellow oil. ${ }^{1} \mathrm{H}$ NMR $\left(400 \mathrm{MHz}, \mathrm{CDCl}_{3}\right) \delta 7.24(\mathrm{dd}, J=7.6$, $1.7 \mathrm{~Hz}, 1 \mathrm{H}), 7.12-7.03(\mathrm{~m}, 3 \mathrm{H}), 6.88(\mathrm{td}, J=7.5,1.2 \mathrm{~Hz}, 1 \mathrm{H}), 6.79-6.70(\mathrm{~m}, 2 \mathrm{H}), 6.63(\mathrm{dd}, J=8.0,1.2 \mathrm{~Hz}$, $1 \mathrm{H}), 5.30(\mathrm{~d}, J=5.7 \mathrm{~Hz}, 1 \mathrm{H}), 4.78(\mathrm{dd}, J=11.4,1.3 \mathrm{~Hz}, 1 \mathrm{H}), 4.38(\mathrm{dd}, J=11.4,1.0 \mathrm{~Hz}, 1 \mathrm{H}), 4.32(\mathrm{td}, J=8.4$, $3.8 \mathrm{~Hz}, 1 \mathrm{H}), 4.08(\mathrm{td}, J=8.5,7.3 \mathrm{~Hz}, 1 \mathrm{H}), 3.85-3.72(\mathrm{~m}, 6 \mathrm{H}), 3.40-3.32(\mathrm{~m}, 1 \mathrm{H}), 2.74(\mathrm{td}, J=9.5,5.7 \mathrm{~Hz}$, $1 \mathrm{H}), 2.46(\mathrm{dtd}, J=12.8,7.2,3.7 \mathrm{~Hz}, 1 \mathrm{H}), 2.28(\mathrm{dq}, J=12.8,8.5 \mathrm{~Hz}, 1 \mathrm{H}), 2.06$ (dddd, $J=12.9,9.3,7.3$, $4.7 \mathrm{~Hz}, 1 \mathrm{H}), 1.85-1.66(\mathrm{~m}, 1 \mathrm{H}) .{ }^{13} \mathrm{C}$ NMR $\left(101 \mathrm{MHz}, \mathrm{CDCl}_{3}\right) \delta 208.9,158.5,152.9,129.1,128.9,127.4$, 127.4, 125.1, 120.6, 115.8, 113.7, 104.5, 101.4, 84.9, 78.0, 67.6, 66.8, 55.2, 49.8, 49.6, 33.1, 26.9. HRMS ESI: Calculated for $\mathrm{C}_{24} \mathrm{H}_{24} \mathrm{NaO}_{4}[\mathrm{M}+\mathrm{Na}]^{+}$399.1572, found 399.1551 (Diff.: 4.13 ppm).

4.3.5. Synthesis of (2S,3R,3a'R,9a'S)-3-(1-(3-fluorophenyl)propa-1,2-dien-1-yl)-2' ,3' ,3a' ,4,5,9a'hexahydro-3H-spiro[furan-2, $4^{\prime}$-furo[2,3-b]chromene] $4 \mathbf{e}$

Compound 4e was prepared following the general procedure using compound $1 \mathbf{e}(50 \mathrm{mg}$, $0.170 \mathrm{mmol}, 1 \mathrm{eq}$.$) , dihydrofuran (119 \mathrm{mg}, 1.70 \mathrm{mmol}, 10$ eq.) and catalyst $\mathbf{A}(7 \mathrm{mg}, 0.009 \mathrm{mmol}$, 0.05 eq.) in 1,4-dioxane $(1 \mathrm{~mL})$. Purification by chromatography on silica gel $\left(8 / 2\right.$ pentane/ $\left.\mathrm{Et}_{2} \mathrm{O}\right)$ afforded compound $4 \mathbf{e}(65 \%, 41 \mathrm{mg}, 0.11 \mathrm{mmol})$ as orange oil. ${ }^{1} \mathrm{H}$ NMR $\left(400 \mathrm{MHz}, \mathrm{CDCl}_{3}\right) \delta 7.28-7.22$ $(\mathrm{m}, 1 \mathrm{H}), 7.18-7.05(\mathrm{~m}, 2 \mathrm{H}), 6.96-6.86(\mathrm{~m}, 2 \mathrm{H}), 6.85-6.75(\mathrm{~m}, 2 \mathrm{H}), 6.60(\mathrm{dd}, J=8.1,1.2 \mathrm{~Hz}, 1 \mathrm{H})$, $5.40(\mathrm{~d}, J=5.8 \mathrm{~Hz}, 1 \mathrm{H}), 4.87(\mathrm{dd}, J=12.0,1.3 \mathrm{~Hz}, 1 \mathrm{H}), 4.52(\mathrm{~d}, J=11.9 \mathrm{~Hz}, 1 \mathrm{H}), 4.31(\mathrm{td}, J=8.4,4.3 \mathrm{~Hz}$, $1 \mathrm{H}), 4.09(\mathrm{q}, J=8.1 \mathrm{~Hz}, 1 \mathrm{H}), 3.89-3.75(\mathrm{~m}, 2 \mathrm{H}), 3.39(\mathrm{dd}, J=8.1,6.8 \mathrm{~Hz}, 1 \mathrm{H}), 2.79(\mathrm{td}, J=9.4,5.8 \mathrm{~Hz}, 1 \mathrm{H})$, $2.50(\mathrm{dtd}, J=12.8,7.5,4.2 \mathrm{~Hz}, 1 \mathrm{H}), 2.38-2.24(\mathrm{~m}, 1 \mathrm{H}), 2.06$ (dddd, $J=12.9,9.3,6.6,5.5 \mathrm{~Hz}, 1 \mathrm{H}), 1.83-1.64$ $(\mathrm{m}, 1 \mathrm{H}) .{ }^{13} \mathrm{C}$ NMR $\left(101 \mathrm{MHz}, \mathrm{CDCl}_{3}\right) \delta 209.2,162.8(\mathrm{~d}, J=244.9 \mathrm{~Hz}), 152.9,139.4(\mathrm{~d}, J=7.6 \mathrm{~Hz})$, $129.6(\mathrm{~d}, J=8.4 \mathrm{~Hz}), 129.1,127.4,125.2,121.8(\mathrm{~d}, J=2.7 \mathrm{~Hz}), 120.9,116.1,113.6(\mathrm{~d}, J=21.2 \mathrm{~Hz})$, $113.5(\mathrm{~d}, J=23.2 \mathrm{~Hz}), 104.9,101.6,84.9,78.8,67.8,66.8,49.5,49.1,33.1,27.0 .{ }^{19} \mathrm{~F}$ NMR $\left(376 \mathrm{MHz}, \mathrm{CDCl}_{3}\right)$ $\delta-113.54-113.72(\mathrm{~m})$. HRMS ESI: Calculated for $\mathrm{C}_{23} \mathrm{H}_{22} \mathrm{FO}_{3}[\mathrm{M}+\mathrm{H}]^{+} 365.1553$, found 365.1546 (Diff.: $0.51 \mathrm{ppm}$ ).

4.3.6. Synthesis of (2S,3R,3a'R,9a'S)-3-(1-(3-bromophenyl)propa-1,2-dien-1-yl)-2', 3',3a',4,5,9a'hexahydro-3H-spiro[furan-2,4'-furo[2,3-b]chromene] $4 \mathbf{f}$

Compound $4 \mathbf{f}$ was prepared following the general procedure using compound $1 \mathrm{f}$ ( $53 \mathrm{mg}$, $0.149 \mathrm{mmol}, 1$ eq.), dihydrofuran (104 mg, $1.49 \mathrm{mmol}, 10$ eq.) and catalyst $\mathbf{A}(7 \mathrm{mg}, 0.009 \mathrm{mmol}$, 0.05 eq.) in 1,4-dioxane $(1 \mathrm{~mL})$. Purification by chromatography on silica gel $\left(8 / 2\right.$ pentane/Et $\left.{ }_{2} \mathrm{O}\right)$ afforded compound $4 \mathrm{f}(70 \%, 45 \mathrm{mg}, 0.104 \mathrm{mmol})$ as an amorphous yellow solid. ${ }^{1} \mathrm{H}$ NMR $(400 \mathrm{MHz}$, $\left.\mathrm{CDCl}_{3}\right) \delta 7.24(\mathrm{dd}, J=7.6,1.7 \mathrm{~Hz}, 1 \mathrm{H}), 7.22-7.18(\mathrm{~m}, 1 \mathrm{H}), 7.12(\mathrm{q}, J=1.4 \mathrm{~Hz}, 1 \mathrm{H}), 7.10-7.00(\mathrm{~m}, 3 \mathrm{H})$, $6.89(\mathrm{td}, J=7.5,1.2 \mathrm{~Hz}, 1 \mathrm{H}), 6.57(\mathrm{dd}, J=8.1,1.2 \mathrm{~Hz}, 1 \mathrm{H}), 5.43(\mathrm{~d}, J=5.8 \mathrm{~Hz}, 1 \mathrm{H}), 4.89(\mathrm{dd}, J=11.9$, $1.5 \mathrm{~Hz}, 1 \mathrm{H}), 4.58(\mathrm{dd}, J=12.0,1.1 \mathrm{~Hz}, 1 \mathrm{H}), 4.29(\mathrm{td}, J=8.4,4.6 \mathrm{~Hz}, 1 \mathrm{H}), 3.78(\mathrm{dd}, J=8.4,6.1 \mathrm{~Hz}, 2 \mathrm{H})$, $3.37(\mathrm{tt}, J=7.2,1.4 \mathrm{~Hz}, 1 \mathrm{H}), 2.79(\mathrm{td}, J=9.4,5.8 \mathrm{~Hz}, 1 \mathrm{H}), 2.55-2.42(\mathrm{~m}, 1 \mathrm{H}), 2.27(\mathrm{dtd}, J=12.8,8.2$, $7.0 \mathrm{~Hz}, 1 \mathrm{H}), 2.12-1.98(\mathrm{~m}, 1 \mathrm{H}), 1.69$ (ddt, $J=12.9,9.7,8.4 \mathrm{~Hz}, 1 \mathrm{H}) .{ }^{13} \mathrm{C}$ NMR $\left(101 \mathrm{MHz}, \mathrm{CDCl}_{3}\right) \delta 209.1$, 152.9, 139.1, 129.6 (3C), 129.1, 127.2, 125.1, 124.8, 122.4, 121.0, 116.1, 105.0, 101.6, 84.9, 78.9, 67.8, 66.7, 
49.4, 48.9, 32.9, 26.9. HRMS ESI: Calculated for $\mathrm{C}_{23} \mathrm{H}_{21} \mathrm{BrNaO}_{3}[\mathrm{M}+\mathrm{Na}]^{+} 447.0572$, found 447.0567 (Diff.: -0.49 ppm).

4.3.7. Synthesis of (2S,3R,3a'R,9a'S)-3-(1-(3-chloro-4-fluorophenyl)propa-1,2-dien-1-yl)-2', $3^{\prime}, 3 a^{\prime}, 4,5,9 a^{\prime}-$ hexahydro-3H-spiro[furan-2, $4^{\prime}$-furo[2,3-b]chromene] $\mathbf{4 g}$

Compound $4 \mathbf{g}$ was prepared following the general procedure using compound $\mathbf{1 g}(52 \mathrm{mg}$, $0.16 \mathrm{mmol}, 1$ eq.), dihydrofuran (112 mg, $1.6 \mathrm{mmol}, 10$ eq.) and catalyst $\mathbf{A}(8 \mathrm{mg}, 0.010 \mathrm{mmol}, 0.05 \mathrm{eq}$.) in 1,4-dioxane $(1 \mathrm{~mL})$. Purification by chromatography on silica gel $\left(8 / 2\right.$ pentane/ $\left./ \mathrm{Et}_{2} \mathrm{O}\right)$ afforded compound $4 \mathrm{~g}(44 \%, 28 \mathrm{mg}, 0.070 \mathrm{mmol})$ as a yellow oil. ${ }^{1} \mathrm{H} \mathrm{NMR}\left(400 \mathrm{MHz}, \mathrm{CDCl}_{3}\right) \delta 7.27-7.21(\mathrm{~m}, 1 \mathrm{H})$, $7.05(\mathrm{ddd}, J=8.1,7.3,1.7 \mathrm{~Hz}, 1 \mathrm{H}), 7.00-6.85(\mathrm{~m}, 4 \mathrm{H}), 6.55(\mathrm{dd}, J=8.0,1.2 \mathrm{~Hz}, 1 \mathrm{H}), 5.48(\mathrm{~d}, J=5.9 \mathrm{~Hz}, 1 \mathrm{H})$, $4.92(\mathrm{dt}, J=11.9,1.2 \mathrm{~Hz}, 1 \mathrm{H}), 4.66(\mathrm{dt}, J=11.8,1.2 \mathrm{~Hz}, 1 \mathrm{H}), 4.28(\mathrm{td}, J=8.4,5.1 \mathrm{~Hz}, 1 \mathrm{H}), 4.07(\mathrm{td}, J=8.2$, $7.1 \mathrm{~Hz}, 1 \mathrm{H}), 3.82-3.75(\mathrm{~m}, 2 \mathrm{H}), 3.35(\mathrm{ddt}, J=7.7,6.2,1.5 \mathrm{~Hz}, 1 \mathrm{H}), 2.82(\mathrm{td}, J=9.4,5.9 \mathrm{~Hz}, 1 \mathrm{H})$, $2.52(\mathrm{dtd}, J=12.8,7.8,5.1 \mathrm{~Hz}, 1 \mathrm{H}), 2.28$ (dddd, $J=13.2,8.4,7.2,6.1 \mathrm{~Hz}, 1 \mathrm{H}), 2.11-1.98(\mathrm{~m}, 1 \mathrm{H})$, $1.68(\mathrm{ddt}, J=12.9,9.5,8.3 \mathrm{~Hz}, 1 \mathrm{H}) .{ }^{13} \mathrm{C} \mathrm{NMR}\left(101 \mathrm{MHz}, \mathrm{CDCl}_{3}\right) \delta 208.9,156.9(\mathrm{~d}, J=256.6 \mathrm{~Hz}), 152.8$, $134.0(\mathrm{~d}, J=3.3 \mathrm{~Hz}), 129.1,128.7,127.1,125.9(\mathrm{~d}, J=7.1 \mathrm{~Hz}), 125.3,121.1,120.6(\mathrm{~d}, J=17.8 \mathrm{~Hz}), 116.1$, $116.0(\mathrm{~d}, J=21.1 \mathrm{~Hz}), 104.8,101.7,84.8,79.1,67.9,66.5,49.2,48.9,32.8,26.9$. HRMS ESI: Calculated for $\mathrm{C}_{23} \mathrm{H}_{21} \mathrm{ClFO}_{3}[\mathrm{M}+\mathrm{H}]^{+}$399.1163, found 399.1165 (Diff.: $-0.61 \mathrm{ppm}$ ).

4.3.8. Synthesis of (2S,3R,3a'R,9a'S)-3-(1-(4-bromo-3-fluorophenyl)propa-1,2-dien-1-yl)-2' , $3^{\prime}, 3 a^{\prime}, 4,5,9 a^{\prime}-$ hexahydro-3H-spiro[furan-2,4'-furo[2,3-b]chromene] $4 \mathrm{~h}$

Compound $4 \mathrm{~h}$ was prepared following the general procedure using compound $\mathbf{1 h}(50 \mathrm{mg}$, $0.152 \mathrm{mmol}, 1$ eq.), dihydrofuran (106 $\mathrm{mg}, 1.52 \mathrm{mmol}, 10$ eq.) and catalyst $\mathbf{A}(6 \mathrm{mg}, 0.008 \mathrm{mmol}$, 0.05 eq.) in 1,4-dioxane ( $1 \mathrm{~mL}$ ). Purification by chromatography on silica gel $\left(8 / 2\right.$ pentane/Et $\left.{ }_{2} \mathrm{O}\right)$ afforded compound $4 \mathrm{~h}(46 \%, 24 \mathrm{mg}, 0.070 \mathrm{mmol})$ as a yellow oil. ${ }^{1} \mathrm{H}$ NMR $\left(400 \mathrm{MHz}, \mathrm{CDCl}_{3}\right) \delta 7.32(\mathrm{dd}, J=8.6$, $7.3 \mathrm{~Hz}, 1 \mathrm{H}), 7.24(\mathrm{dd}, J=7.5,1.8 \mathrm{~Hz}, 1 \mathrm{H}), 7.07(\mathrm{ddd}, J=8.1,7.3,1.7 \mathrm{~Hz}, 1 \mathrm{H}), 6.88(\mathrm{td}, J=7.5,1.2 \mathrm{~Hz}, 1 \mathrm{H})$, 6.84-6.75 (m, 2H), $6.58(\mathrm{dd}, J=8.1,1.2 \mathrm{~Hz}, 1 \mathrm{H}), 5.45(\mathrm{~d}, J=5.9 \mathrm{~Hz}, 1 \mathrm{H}), 4.91(\mathrm{dd}, J=12.1,1.4 \mathrm{~Hz}, 1 \mathrm{H})$, $4.59(\mathrm{dd}, J=12.2,1.0 \mathrm{~Hz}, 1 \mathrm{H}), 4.30(\mathrm{td}, J=8.4,4.7 \mathrm{~Hz}, 1 \mathrm{H}), 4.08(\mathrm{q}, J=7.9 \mathrm{~Hz}, 1 \mathrm{H}), 3.78(\mathrm{dd}, J=8.4$, $6.1 \mathrm{~Hz}, 2 \mathrm{H}), 3.35(\mathrm{t}, J=7.1 \mathrm{~Hz}, 1 \mathrm{H}), 2.81(\mathrm{td}, J=9.3,5.9 \mathrm{~Hz}, 1 \mathrm{H}), 2.51(\mathrm{dtd}, J=12.5,7.7,4.7 \mathrm{~Hz}, 1 \mathrm{H})$, $2.27(\mathrm{dq}, J=12.9,7.6 \mathrm{~Hz}, 1 \mathrm{H}), 2.05(\mathrm{ddt}, J=12.7,9.4,6.1 \mathrm{~Hz}, 1 \mathrm{H}), 1.69(\mathrm{dq}, J=13.0,8.5 \mathrm{~Hz}, 1 \mathrm{H}) .{ }^{13} \mathrm{C} \mathrm{NMR}$ $\left(101 \mathrm{MHz}, \mathrm{CDCl}_{3}\right) \delta 209.1,158.8(\mathrm{~d}, J=246.4 \mathrm{~Hz}), 152.9,138.6(\mathrm{~d}, J=6.9 \mathrm{~Hz}), 132.9(\mathrm{~d}, J=1.0 \mathrm{~Hz})$, 129.2, 127.3, 125.4, 122.9 (d, $J=3.2 \mathrm{~Hz}), 121.1,116.2,114.5(\mathrm{~d}, J=23.5 \mathrm{~Hz}), 106.8(\mathrm{~d}, J=21.2 \mathrm{~Hz})$, $104.86(\mathrm{~d}, J=2.2 \mathrm{~Hz}), 101.7,84.9,79.3,67.9,66.6,49.4,48.7,32.9,27.0$. HRMS ESI: Calculated for $\mathrm{C}_{23} \mathrm{H}_{20} \mathrm{BrFNaO}_{3}[\mathrm{M}+\mathrm{Na}]^{+}$465.0478, found 465.0464 (Diff.: 0.65 ppm).

4.3.9. Synthesis of (2S,3R,3a'R,9a'S)-3-(1-(4-chlorophenyl)propa-1,2-dien-1-yl)-2', 3', 3a', 4,5,9a'hexahydro-3H-spiro[furan-2,4'-furo[2,3-b]chromene] $4 \mathbf{i}$

Compound $4 \mathbf{i}$ was prepared following the general procedure using compound $1 \mathbf{i}(50 \mathrm{mg}$, $0.161 \mathrm{mmol}, 1$ eq.), dihydrofuran $(113 \mathrm{mg}, 1.61 \mathrm{mmol}, 10$ eq.) and catalyst $\mathbf{A}(8 \mathrm{mg}, 0.010 \mathrm{mmol}$, 0.05 eq.) in 1,4-dioxane $(1 \mathrm{~mL})$. Purification by chromatography on silica gel $\left(8 / 2\right.$ pentane $\left./ \mathrm{Et}_{2} \mathrm{O}\right)$ afforded compound $4 \mathbf{i}(28 \%, 17 \mathrm{mg}, 0.045 \mathrm{mmol})$ as a colorless oil. ${ }^{1} \mathrm{H}$ NMR $\left(400 \mathrm{MHz}, \mathrm{CDCl}_{3}\right) \delta 7.25(\mathrm{dd}, J=7.6$, $1.7 \mathrm{~Hz}, 1 \mathrm{H}), 7.16-7.11(\mathrm{~m}, 2 \mathrm{H}), 7.09-7.01(\mathrm{~m}, 3 \mathrm{H}), 6.88(\mathrm{td}, J=7.5,1.2 \mathrm{~Hz}, 1 \mathrm{H}), 6.59(\mathrm{dd}, J=8.1,1.2 \mathrm{~Hz}$, $1 \mathrm{H}), 5.38(\mathrm{~d}, J=5.8 \mathrm{~Hz}, 1 \mathrm{H}), 4.86(\mathrm{dd}, J=11.9,1.4 \mathrm{~Hz}, 1 \mathrm{H}), 4.51(\mathrm{dd}, J=11.9,1.1 \mathrm{~Hz}, 1 \mathrm{H}), 4.31(\mathrm{td}, J=8.4$, $4.3 \mathrm{~Hz}, 1 \mathrm{H}), 4.08(\mathrm{q}, J=8.0 \mathrm{~Hz}, 1 \mathrm{H}), 3.84-3.73(\mathrm{~m}, 2 \mathrm{H}), 3.37(\mathrm{tt}, J=7.3,1.3 \mathrm{~Hz}, 1 \mathrm{H}), 2.78(\mathrm{td}, J=9.4$, $5.8 \mathrm{~Hz}, 1 \mathrm{H}), 2.50(\mathrm{dtd}, J=12.7,7.5,4.3 \mathrm{~Hz}, 1 \mathrm{H}), 2.28(\mathrm{dtd}, J=12.8,8.3,7.4 \mathrm{~Hz}, 1 \mathrm{H}), 2.05(\mathrm{dddd}, J=12.9$, 9.3, 6.7, $5.4 \mathrm{~Hz}, 1 \mathrm{H}), 1.79-1.60(\mathrm{~m}, 1 \mathrm{H}) .{ }^{13} \mathrm{C} \mathrm{NMR}\left(101 \mathrm{MHz}, \mathrm{CDCl}_{3}\right) \delta 209.1,152.9,135.4,132.4,129.1$, 128.4, 127.7, 127.4, 125.3, 121.0, 116.2, 104.9, 101.6, 84.9, 78.7, 67.9, 66.8, 49.5, 49.1, 33.1, 27.0. HRMS ESI: Calculated for $\mathrm{C}_{23} \mathrm{H}_{22} \mathrm{ClO}_{3}[\mathrm{M}+\mathrm{H}]^{+} 381.1257$, found 381.1255 (Diff.: -0.64 ppm). 
4.3.10. Synthesis of (2S,3R,3a'R,9a'S)-3-(1-(4-(trifluoromethyl)phenyl)propa-1,2-dien-1-yl)$2^{\prime}, 3^{\prime}, 3 a^{\prime}, 4,5,9 a^{\prime}$-hexahydro-3H-spiro[furan-2,4'-furo[2,3-b]chromene] 4 j

Compound $\mathbf{4 j}$ was prepared following the general procedure using compound $\mathbf{1 j}$ (50 $\mathrm{mg}$, $0.145 \mathrm{mmol}, 1$ eq.), dihydrofuran (102 mg, $1.45 \mathrm{mmol}, 10$ eq.) and catalyst $\mathbf{A}$ (6 mg, $0.007 \mathrm{mmol}$, 0.05 eq.) in 1,4-dioxane $(1 \mathrm{~mL})$. Purification by chromatography on silica gel $\left(8 / 2\right.$ pentane/Et $\left.{ }_{2} \mathrm{O}\right)$ afforded compound $4 \mathbf{j}(44 \%, 26 \mathrm{mg}, 0.064 \mathrm{mmol})$ as an amorphous yellow solid. ${ }^{1} \mathrm{H} \mathrm{NMR}\left(400 \mathrm{MHz}, \mathrm{CDCl}_{3}\right)$ 反 7.42-7.36 (m, 2H), 7.30-7.23 (m, 1H), $7.19(\mathrm{~d}, J=8.1 \mathrm{~Hz}, 2 \mathrm{H}), 7.03(\mathrm{ddd}, J=8.0,7.3,1.7 \mathrm{~Hz}, 1 \mathrm{H})$, $6.87(\mathrm{td}, J=7.5,1.2 \mathrm{~Hz}, 1 \mathrm{H}), 6.53(\mathrm{dd}, J=8.1,1.2 \mathrm{~Hz}, 1 \mathrm{H}), 5.43(\mathrm{~d}, J=6.0 \mathrm{~Hz}, 1 \mathrm{H}), 5.02-4.82(\mathrm{~m}, 1 \mathrm{H})$, $4.62(\mathrm{~d}, J=12.1 \mathrm{~Hz}, 1 \mathrm{H}), 4.31(\mathrm{td}, J=8.4,4.7 \mathrm{~Hz}, 1 \mathrm{H}), 4.10(\mathrm{q}, J=8.0 \mathrm{~Hz}, 1 \mathrm{H}), 3.91-3.68(\mathrm{~m}, 2 \mathrm{H}), 3.57-3.35$ $(\mathrm{m}, 1 \mathrm{H}), 2.82(\mathrm{td}, J=9.4,5.9 \mathrm{~Hz}, 1 \mathrm{H}), 2.54(\mathrm{dtd}, J=12.6,7.7,4.7 \mathrm{~Hz}, 1 \mathrm{H}), 2.44-2.23(\mathrm{~m}, 1 \mathrm{H}), 2.13-1.97$ $(\mathrm{m}, 1 \mathrm{H}), 1.69(\mathrm{ddt}, J=12.9,9.5,8.4 \mathrm{~Hz}, 1 \mathrm{H}) .{ }^{13} \mathrm{C} \mathrm{NMR}\left(101 \mathrm{MHz}, \mathrm{CDCl}_{3}\right) \delta 209.6,152.9,140.7,129.2$, $128.7(q, J=32.4 \mathrm{~Hz}), 127.3,126.6,125.4,125.1(\mathrm{q}, J=3.8 \mathrm{~Hz}), 124.4(\mathrm{q}, J=273.7 \mathrm{~Hz}), 121.1,116.3,105.3$, 101.7, 85.0, 79.0, 67.9, 66.7, 49.4, 48.7, 33.0, 27.0. $\left.{ }^{19} \mathrm{~F} \mathrm{NMR} \mathrm{(376} \mathrm{MHz,} \mathrm{CDCl}_{3}\right) \delta-62.48$ (s). HRMS ESI: Calculated for $\mathrm{C}_{24} \mathrm{H}_{21} \mathrm{~F}_{3} \mathrm{NaO}_{3}[\mathrm{M}+\mathrm{Na}]^{+} 437.1340$, found 437.1336 (Diff.: $-0.31 \mathrm{ppm}$ ).

4.3.11. Synthesis of (2S,3R,3a'R,9a'S)-3-(1-(4-nitrophenyl)propa-1,2-dien-1-yl)-2' ,3',3a' ,4,5,9a' hexahydro-3H-spiro[furan-2, $4^{\prime}$-furo[2,3-b]chromene] $4 \mathbf{k}$

Compound $4 \mathbf{k}$ was prepared following the general procedure using compound 1k (50 $\mathrm{mg}$, $0.205 \mathrm{mmol}, 1$ eq.), dihydrofuran (167 mg, $2.05 \mathrm{mmol}, 10$ eq.) and catalyst A (9 mg, $0.012 \mathrm{mmol}$, 0.05 eq.) in 1,4-dioxane $(1 \mathrm{~mL})$. Purification by chromatography on silica gel $\left(8 / 2\right.$ pentane/Et $\left.{ }_{2} \mathrm{O}\right)$ afforded compound $4 \mathbf{k}(60 \%, 48 \mathrm{mg}, 0.12 \mathrm{mmol})$ as an orange oil. ${ }^{1} \mathrm{H}$ NMR $\left(400 \mathrm{MHz}, \mathrm{CDCl}_{3}\right)$ $\delta 7.98(\mathrm{~d}, J=9.0 \mathrm{~Hz}, 2 \mathrm{H}), 7.26(\mathrm{dd}, J=7.5,1.7 \mathrm{~Hz}, 1 \mathrm{H}), 7.20(\mathrm{~d}, J=8.9 \mathrm{~Hz}, 2 \mathrm{H}), 7.06-6.97(\mathrm{~m}, 1 \mathrm{H})$, $6.86(\mathrm{td}, J=7.5,1.2 \mathrm{~Hz}, 1 \mathrm{H}), 6.49(\mathrm{dd}, J=8.0,1.2 \mathrm{~Hz}, 1 \mathrm{H}), 5.52(\mathrm{~d}, J=6.1 \mathrm{~Hz}, 1 \mathrm{H}), 5.04(\mathrm{dd}, J=12.7$, $1.6 \mathrm{~Hz}, 1 \mathrm{H}), 4.79(\mathrm{dd}, J=12.7,1.2 \mathrm{~Hz}, 1 \mathrm{H}), 4.31(\mathrm{td}, J=8.5,5.3 \mathrm{~Hz}, 1 \mathrm{H}), 4.09(\mathrm{td}, J=8.4,6.9 \mathrm{~Hz}, 1 \mathrm{H})$, $3.77(\mathrm{ddd}, J=8.5,6.2,3.4 \mathrm{~Hz}, 2 \mathrm{H}), 3.63-3.45(\mathrm{~m}, 1 \mathrm{H}), 2.88(\mathrm{td}, J=9.3,6.1 \mathrm{~Hz}, 1 \mathrm{H}), 2.59(\mathrm{dtd}, J=13.1$, 8.0, 5.3 Hz, 1H), 2.40-2.28 (m, 1H), 2.04 (dddd, $J=12.6,9.4,7.2,5.2 \mathrm{~Hz}, 1 \mathrm{H}), 1.69(\mathrm{dq}, J=13.1,8.6 \mathrm{~Hz}$, 1H). ${ }^{13} \mathrm{C} \mathrm{NMR}\left(101 \mathrm{MHz}, \mathrm{CDCl}_{3}\right) \delta 210.0,152.7,146.2,143.7,129.2,127.2,126.9,125.6,123.3,121.3$, 116.3, 105.8, 101.8, 84.8, 79.6, 67.9, 66.4, 49.1, 47.9, 32.7, 26.9. HRMS ESI: Calculated for $\mathrm{C}_{23} \mathrm{H}_{21} \mathrm{NNaO}_{5}$ $[\mathrm{M}+\mathrm{Na}]^{+} 414.1317$, found 414.1309 (Diff.: 0.35 ppm).

4.3.12. Synthesis of (2S,3R,3a'R,9a'S)-3-(1-(3-nitrophenyl)propa-1,2-dien-1-yl)-2' , $3^{\prime}, 3 a^{\prime}, 4,5,9 a^{\prime}-$ hexahydro-3H-spiro[furan-2,4'-furo[2,3-b]chromene] 41

Compound 41 was prepared following the general procedure using compound 11 (51 mg, $0.159 \mathrm{mmol}, 1$ eq.), dihydrofuran (111 mg, $1.59 \mathrm{mmol}, 10$ eq.) and catalyst A (8 mg, $0.010 \mathrm{mmol}$, 0.05 eq.) in 1,4-dioxane $(1 \mathrm{~mL})$. Purification by chromatography on silica gel $\left(8 / 2\right.$ pentane/Et $\left.\mathrm{E}_{2} \mathrm{O}\right)$ afforded compound $41(34 \%, 21 \mathrm{mg}, 0.054 \mathrm{mmol})$ as an orange oil. ${ }^{1} \mathrm{H}$ NMR $\left(400 \mathrm{MHz}, \mathrm{CDCl}_{3}\right)$ $\delta 7.90(\mathrm{ddd}, J=8.0,2.3,1.2 \mathrm{~Hz}, 1 \mathrm{H}), 7.81(\mathrm{t}, J=2.0 \mathrm{~Hz}, 1 \mathrm{H}), 7.34(\mathrm{dt}, J=7.8,1.5 \mathrm{~Hz}, 1 \mathrm{H}), 7.31-7.23$ $(\mathrm{m}, 2 \mathrm{H}), 7.04-6.92(\mathrm{~m}, 1 \mathrm{H}), 6.87(\mathrm{td}, J=7.4,1.3 \mathrm{~Hz}, 1 \mathrm{H}), 6.42(\mathrm{dd}, J=8.0,1.3 \mathrm{~Hz}, 1 \mathrm{H}), 5.53(\mathrm{~d}, J=6.0 \mathrm{~Hz}$, $1 \mathrm{H}), 5.03(\mathrm{dd}, J=12.2,1.7 \mathrm{~Hz}, 1 \mathrm{H}), 4.83(\mathrm{dd}, J=12.3,1.4 \mathrm{~Hz}, 1 \mathrm{H}), 4.30(\mathrm{td}, J=8.4,5.5 \mathrm{~Hz}, 1 \mathrm{H})$, $4.10(\mathrm{td}, J=8.4,6.8 \mathrm{~Hz}, 1 \mathrm{H}), 3.84-3.70(\mathrm{~m}, 2 \mathrm{H}), 3.51(\mathrm{ddt}, J=7.2,5.4,1.6 \mathrm{~Hz}, 1 \mathrm{H}), 2.88(\mathrm{td}, J=9.3$, $6.0 \mathrm{~Hz}, 1 \mathrm{H}), 2.68-2.50(\mathrm{~m}, 1 \mathrm{H}), 2.43-2.28(\mathrm{~m}, 1 \mathrm{H}), 2.04(\mathrm{dddd}, J=12.7,9.4,7.3,5.0 \mathrm{~Hz}, 1 \mathrm{H}), 1.76-1.60$ $(\mathrm{m}, 1 \mathrm{H}) .{ }^{13} \mathrm{C}$ NMR $\left(101 \mathrm{MHz}, \mathrm{CDCl}_{3}\right) \delta 209.2,152.8,148.1,138.7,132.3,129.1,128.8,127.1,125.6,125.5$, 121.4, 121.3, 116.2, 105.5, 101.8, 84.9, 79.7, 68.0, 66.5, 49.1, 48.3, 32.6, 26.9. HRMS ESI: Calculated for $\mathrm{C}_{23} \mathrm{H}_{21} \mathrm{NNaO}_{5}[\mathrm{M}+\mathrm{Na}]^{+} 414.1317$, found 414.1296 (Diff.: 4.40 ppm).

4.3.13. Synthesis of (2S,3R,3a' R,9a'S)-3-(1-(thiophen-2-yl)propa-1,2-dien-1-yl)-2' , 3' ,3a' ,4,5,9a' hexahydro-3H-spiro[furan-2,4'-furo[2,3-b]chromene] $4 \mathrm{~m}$

Compound $4 \mathrm{~m}$ was prepared following the general procedure using compound $1 \mathrm{~m}(50 \mathrm{mg}$, $0.177 \mathrm{mmol}, 1$ eq.), dihydrofuran (124 mg, $1.77 \mathrm{mmol}, 10$ eq.) and catalyst A (7 mg, $0.09 \mathrm{mmol}$, 0.05 eq.) in 1,4-dioxane $(1 \mathrm{~mL})$. Purification by chromatography on silica gel $\left(8 / 2\right.$ pentane/Et $\left.{ }_{2} \mathrm{O}\right)$ 
afforded compound $4 \mathrm{~m}(65 \%, 41 \mathrm{mg}, 0.117 \mathrm{mmol})$ as an amorphous yellow solid. ${ }^{1} \mathrm{H}$ NMR $(400 \mathrm{MHz}$, $\left.\mathrm{CDCl}_{3}\right) \delta 7.24(\mathrm{dd}, J=7.6,1.7 \mathrm{~Hz}, 1 \mathrm{H}), 7.15-7.05(\mathrm{~m}, 2 \mathrm{H}), 6.93-6.79(\mathrm{~m}, 3 \mathrm{H}), 6.66(\mathrm{dd}, J=8.1,1.2 \mathrm{~Hz}$, $1 \mathrm{H}), 5.54(\mathrm{~d}, J=5.7 \mathrm{~Hz}, 1 \mathrm{H}), 4.89(\mathrm{~d}, J=12.3 \mathrm{~Hz}, 1 \mathrm{H}), 4.48(\mathrm{dd}, J=12.3,0.9 \mathrm{~Hz}, 1 \mathrm{H}), 4.30(\mathrm{td}, J=8.5$, $3.9 \mathrm{~Hz}, 1 \mathrm{H}), 4.14-4.00(\mathrm{~m}, 1 \mathrm{H}), 3.92-3.75(\mathrm{~m}, 2 \mathrm{H}), 3.42-3.25(\mathrm{~m}, 1 \mathrm{H}), 2.83(\mathrm{td}, J=9.5,5.7 \mathrm{~Hz}, 1 \mathrm{H})$, $2.47(\mathrm{dtd}, J=12.8,7.4,3.9 \mathrm{~Hz}, 1 \mathrm{H}), 2.25(\mathrm{dq}, J=12.8,8.4 \mathrm{~Hz}, 1 \mathrm{H}), 2.08(\mathrm{dddd}, J=12.8,9.2,7.2,4.9 \mathrm{~Hz}$, 1H), $1.72(\mathrm{ddt}, J=12.9,9.8,8.5 \mathrm{~Hz}, 1 \mathrm{H}) .{ }^{13} \mathrm{C}$ NMR $\left(101 \mathrm{MHz}, \mathrm{CDCl}_{3}\right) \delta 208.4,153.0,141.3,129.1,127.5$, 127.3, 124.9, 124.5, 123.0, 120.8, 116.1, 101.7, 100.8, 84.9, 79.5, 67.8, 66.8, 51.1, 49.6, 32.9, 27.1. HRMS ESI: Calculated for $\mathrm{C}_{21} \mathrm{H}_{21} \mathrm{SO}_{3}[\mathrm{M}+\mathrm{H}]^{+} 353.1211$, found 353.1196 (Diff.: 2.77 ppm).

4.3.14. Synthesis of (2S,3R,3a'R,9a'S)-3-(1-(benzo[b]thiophen-2-yl)propa-1,2-dien-1-yl)-2' ,3',3a' ,4,5,9a'hexahydro-3H-spiro[furan-2,4'-furo[2,3-b]chromene] $4 \mathbf{n}$

Compound 4i was prepared following the general procedure using compound $1 \mathbf{i}$ (50 $\mathrm{mg}$, $0.150 \mathrm{mmol}, 1$ eq.), dihydrofuran (105 $\mathrm{mg}, 1.50 \mathrm{mmol}, 10$ eq.) and catalyst $\mathbf{A}(6 \mathrm{mg}, 0.008 \mathrm{mmol}$, 0.05 eq.) in 1,4-dioxane ( $1 \mathrm{~mL})$. Purification by chromatography on silica gel ( $8 / 2$ pentane/Et $\left.{ }_{2} \mathrm{O}\right)$ afforded compound $4 \mathbf{i}(68 \%, 41 \mathrm{mg}, 0.102 \mathrm{mmol})$ as an orange oil. ${ }^{1} \mathrm{H}$ NMR $\left(400 \mathrm{MHz}, \mathrm{CDCl}_{3}\right) \delta 7.7-7.6(\mathrm{~m}, 2 \mathrm{H})$, 7.3-7.2 (m, 3H), 7.2-7.0 (m, 2H), $6.9(\mathrm{td}, J=7.5,1.2 \mathrm{~Hz}, 1 \mathrm{H}), 6.7(\mathrm{dd}, J=8.0,1.2 \mathrm{~Hz}, 1 \mathrm{H}), 5.6(\mathrm{~d}, J=5.8 \mathrm{~Hz}$, $1 \mathrm{H}), 5.0(\mathrm{dt}, J=12.8,1.1 \mathrm{~Hz}, 1 \mathrm{H}), 4.5(\mathrm{dt}, J=12.8,1.0 \mathrm{~Hz}, 1 \mathrm{H}), 4.3(\mathrm{td}, J=8.5,3.6 \mathrm{~Hz}, 1 \mathrm{H}), 4.1(\mathrm{td}, J=8.6$, $7.5 \mathrm{~Hz}, 1 \mathrm{H}), 4.0-3.7(\mathrm{~m}, 2 \mathrm{H}), 3.4(\mathrm{dd}, J=8.4,7.1 \mathrm{~Hz}, 1 \mathrm{H}), 2.9(\mathrm{td}, J=9.4,5.8 \mathrm{~Hz}, 1 \mathrm{H}), 2.5(\mathrm{dtd}, J=12.8$, 7.3, 3.6 Hz, 1H), 2.4-2.2 (m, 1H), 2.2-2.0 (m, 1H), $1.7(\mathrm{ddt}, J=12.9,9.7,8.4 \mathrm{~Hz}, 1 \mathrm{H}) .{ }^{13} \mathrm{C}$ NMR $(101 \mathrm{MHz}$, $\left.\mathrm{CDCl}_{3}\right) \delta 209.3,153.0,141.9,140.4,139.4,129.2,127.5,125.2,124.3,124.2,123.3,122.0,121.0,119.2,116.3$, $101.9,101.4,85.0,80.0,67.8,66.8,50.1,49.7,33.0,27.2$. HRMS ESI: Calculated for $\mathrm{C}_{25} \mathrm{H}_{23} \mathrm{SO}_{3}[\mathrm{M}+\mathrm{H}]^{+}$ 403.1368, found 403.1354 (Diff.: 1.64 ppm).

Procedures for the synthesis of the substrates and the products and full characterization of final products can be found in the Supplementary Materials.

Supplementary Materials: The supplementary materials are available online.

Author Contributions: Conceptualization, G.B.; methodology, M.R., N.B. and R.G.; formal analysis, M.R., N.B. and R.G.; writing—original draft preparation, G.B.; writing—review and editing, G.B.; supervision, G.B. and P.W.; project administration, G.B. All authors have read and agreed to the published version of the manuscript.

Funding: University of Strasbourg Institute for Advanced Study (USIAS), the Chemistry of Complex Systems LabEx and the Frontier Research in Chemistry Foundation.

Acknowledgments: The authors gratefully acknowledge the support of the University of Strasbourg Institute for Advanced Study (USIAS), the Chemistry of Complex Systems LabEx and the Frontier Research in Chemistry Foundation. We sincerely thank the leading committee of the Laboratoire d'Innovation Thérapeutique (UMR 7200) for its technical support.

Conflicts of Interest: The authors declare no conflict of interest.

\section{References}

1. Kam, T.-S.; Choo, Y.-M. Macrodasine A, a Novel Macroline Derivative Incorporating Fused Spirocyclic Tetrahydrofuran Rings Containing a Spiroacetal Moiety. Tetrahedron Lett. 2003, 44, 8787-8789. [CrossRef]

2. Tan, S.-J.; Robinson, W.T.; Komiyama, K.; Kam, T.-S. Macrodasines A-G, Macroline Indole Alkaloids Incorporating Fused Spirocyclic Tetrahydrofuran-Tetrahydrofuran and Tetrahydrofuran-Tetrahydropyran Rings. Tetrahedron 2011, 67, 3830-3838. [CrossRef]

3. Lin, Y.; Wu, X.; Feng, S.; Jiang, G.; Luo, J.; Zhou, S.; Vrijmoed, L.L.P.; Jones, E.B.G.; Krohn, K.; Steingröver, K.; et al. Five Unique Compounds: Xyloketals from Mangrove Fungus Xylaria Sp. from the South China Sea Coast. J. Org. Chem. 2001, 66, 6252-6256. [CrossRef]

4. Wang, W.; Zeng, Y.H.; Osman, K.; Shinde, K.; Rahman, M.; Gibbons, S.; Mu, Q. Norlignans, Acylphloroglucinols, and a Dimeric Xanthone from Hypericum Chinense. J. Nat. Prod. 2010, 73, 1815-1820. [CrossRef] 
5. Trisuwan, K.; Rukachaisirikul, V.; Sukpondma, Y.; Phongpaichit, S.; Preedanon, S.; Sakayaroj, J. Lactone Derivatives from the Marine-Derived Fungus Penicillium Sp. PSU-F44. Chem. Pharm. Bull. 2009, 57, 1100-1102. [CrossRef]

6. Blond, G.; Gulea, M.; Mamane, V. Recent Contributions to Hetero Diels-Alder Reactions. Curr. Org. Chem. 2016, 20, 2161-2210. [CrossRef]

7. Brachet, E.; Belmont, P. Inverse Electron Demand Diels-Alder (IEDDA) Reactions: Synthesis of Heterocycles and Natural Products Along with Bioorthogonal and Material Sciences Applications. COC 2016, 20, 2136-2160. [CrossRef]

8. Pałasz, A. Recent Advances in Inverse-Electron-Demand Hetero-Diels-Alder Reactions of 1-Oxa-1,3-Butadienes. Top. Curr. Chem. 2016, 374, 1-37. [CrossRef] [PubMed]

9. Ishihara, K.; Sakakura, A. 5.10 Hetero-Diels-Alder Reactions. In Comprehensive Organic Synthesis II; Elsevier: Oxford, UK, 2014; pp. 409-465.

10. Sogani, N.; Bansal, R. Catalytic Hetero-Diels-Alder Reaction of the Carbonyl Compounds. CCAT 2017, 6, 3-24. [CrossRef]

11. Skrzyńska, A.; Frankowski, S.; Albrecht, Ł. Cyclic 1-Azadienes in the Organocatalytic Inverse-Electron-Demand Aza-Diels-Alder Cycloadditions. Asian J. Org. Chem. 2020. [CrossRef]

12. Han, Z.-Y.; Chen, D.-F.; Wang, Y.-Y.; Guo, R.; Wang, P.-S.; Wang, C.; Gong, L.-Z. Hybrid Metal/Organo Relay Catalysis Enables Enynes To Be Latent Dienes for Asymmetric Diels-Alder Reaction. J. Am. Chem. Soc. 2012, 134, 6532-6535. [CrossRef] [PubMed]

13. Yan, J.; Tay, G.L.; Neo, C.; Lee, B.R.; Chan, P.W.H. Gold-Catalyzed Cycloisomerization and Diels-Alder Reaction of 1,6-Diyne Esters with Alkenes and Diazenes to Hydronaphthalenes and -Cinnolines. Org. Lett. 2015, 17, 4176-4179. [CrossRef]

14. Du, Y.-L.; Zhao, F.; Han, Z.-Y.; Gong, L.-Z. Chiral Gold Complex Catalyzed Tandem Dehydrative Cyclization/Hetero-Diels-Alder Reaction. Synthesis 2016, 49, 151-158.

15. Wang, X.; Yao, Z.; Dong, S.; Wei, F.; Wang, H.; Xu, Z. Synthesis of Fused Bicyclic Aminals through Sequential Gold/Lewis Acid Catalysis. Org. Lett. 2013, 15, 2234-2237. [CrossRef] [PubMed]

16. Wang, X.; Dong, S.; Yao, Z.; Feng, L.; Daka, P.; Wang, H.; Xu, Z. Synthesis of Spiroaminals and Spiroketals with Bimetallic Relay Catalysis. Org. Lett. 2014, 16, 22-25. [CrossRef] [PubMed]

17. Zhang, S.; Xu, Z.; Jia, J.; Tung, C.-H.; Xu, Z. Synthesis of Spiroaminals by Bimetallic Au/Sc Relay Catalysis: TMS as a Traceless Controlling Group. Chem. Commun. 2014, 50, 12084-12087. [CrossRef]

18. Li, J.; Lin, L.; Hu, B.; Lian, X.; Wang, G.; Liu, X.; Feng, X. Bimetallic Gold(I)/Chiral N, $N^{\prime}$-Dioxide Nickel(II) Asymmetric Relay Catalysis: Chemo- and Enantioselective Synthesis of Spiroketals and Spiroaminals. Angew. Chem. Int. Ed. 2016, 55, 6075-6078. [CrossRef] [PubMed]

19. Gong, J.; Wan, Q.; Kang, Q. Gold(I)/Chiral Rh(III) Lewis Acid Relay Catalysis Enables Asymmetric Synthesis of Spiroketals and Spiroaminals. Angew. Chem. Int. Ed. 2018, 360, 4031-4036. [CrossRef]

20. Hu, B.; Li, J.; Cao, W.; Lin, Q.; Yang, J.; Lin, L.; Liu, X.; Feng, X. Asymmetric Synthesis of Fused Bicyclic $\mathrm{N}, \mathrm{O}$ - and $\mathrm{O}, \mathrm{O}$-Acetals via Cascade Reaction by Gold(I)/N,N'-Dioxide-Nickel(II) Bimetallic Relay Catalysis. Adv. Synth. Catal. 2018, 360, 2831-2835. [CrossRef]

21. Wang, C.-S.; Cheng, Y.-C.; Zhou, J.; Mei, G.-J.; Wang, S.-L.; Shi, F. Metal-Catalyzed Oxa-[4+2] Cyclizations of Quinone Methides with Alkynyl Benzyl Alcohols. J. Org. Chem. 2018, 83, 13861-13873. [CrossRef]

22. Wang, B.; Liang, M.; Tang, J.; Deng, Y.; Zhao, J.; Sun, H.; Tung, C.-H.; Jia, J.; Xu, Z. Gold/Lewis Acid Catalyzed Cycloisomerization/Diastereoselective [3 + 2] Cycloaddition Cascade: Synthesis of Diverse Nitrogen-Containing Spiro Heterocycles. Org. Lett. 2016, 18, 4614-4617. [CrossRef]

23. Arto, T.; Fañanás, F.J.; Rodríguez, F. Gold(I)-Catalyzed Generation of the Two Components of a Formal [4 + 2] Cycloaddition Reaction for the Synthesis of Tetracyclic Pyrano[2,3,4-de ]Chromenes. Angew. Chem. Int. Ed. 2016, 55, 7218-7221. [CrossRef] [PubMed] 
24. Pertschi, R.; Wagner, P.; Ghosh, N.; Gandon, V.; Blond, G. Gold(I)-Catalyzed Synthesis of Furopyrans: Insight into Hetero-Diels-Alder Reactions. Org. Lett. 2019, 21, 6084-6088. [CrossRef]

25. Wagner, P.; Ghosh, N.; Gandon, V.; Blond, G. Solvent Effect in Gold(I)-Catalyzed Domino Reaction : Access to Furopyrans. Org. Lett. 2020, 22, 7333-7337. [CrossRef] [PubMed]

Sample Availability: Samples of the compounds $4 \mathbf{a}-\mathbf{4 n}$ and $5 \mathbf{a}$ are available from the authors.

Publisher's Note: MDPI stays neutral with regard to jurisdictional claims in published maps and institutional affiliations.

(C) 2020 by the authors. Licensee MDPI, Basel, Switzerland. This article is an open access article distributed under the terms and conditions of the Creative Commons Attribution (CC BY) license (http://creativecommons.org/licenses/by/4.0/). 